\title{
FORMATION OF P CYGNI LINE PROFILES IN RELATIVISTICALLY EXPANDING ATMOSPHERES
}

\author{
DAMIEN HUTSEMÉKERS \\ European Southern Observatory, La Silla, Chile \\ AND \\ JEAN SURDEJ ${ }^{1}$ \\ Institut d'Astrophysique, Liège \\ Received 1989 November 10; accepted 1990 March 28
}

\begin{abstract}
The P Cygni line profiles observed in the spectrum of broad absorption-line quasars, supernovae, etc., are sometimes characterized by very large Doppler velocities. In order to interpret more accurately such profiles, we have generalized the Sobolev theory for the transfer of line radiation to the case of the special relativity.

Considering spherically symmetric expanding envelopes, we first discuss the deformations suffered by the surfaces of equal frequency when the velocities become comparable to that of light. For a single line formed in an atmosphere with a monotonic velocity field, we note the possible appearance of distant interactions at very large expansion velocities, a relativistic effect which may be of considerable importance when evaluating the amplitude of radiative forces.

Following a probabilistic formalism, we subsequently establish the expression of the source function and that of the line profile. We find that the relativistic P Cygni line profiles are significantly different from those computed in the framework of the classical theory: for increasing values of the terminal wind velocity, the red emission wing becomes definitely narrower than the blue one while the line center emission increases. These modifications are mainly due to the redistribution of the scattered line photons in a frequency interval which is no longer symmetrical, in accordance with the relativistic expression of the Doppler effect.

We finally study the first order moment of unsaturated $\mathbf{P}$ Cygni line profiles and show that, in the relativistic case, this moment is still directly proportional to the mass-loss rate. For the case of slightly and strongly saturated line profiles, we compute the first-order moment curves of growth which, after normalization, are also found to be little dependent on the relativistic corrections and very useful for the determination of mass-loss rates.
\end{abstract}

Subject headings: line profiles - quasars - radiative transfer - relativity - stars: supernovae

\section{INTRODUCTION}

P Cygni line profiles characterized by relativistic Doppler velocities are currently observed in the spectrum of astronomical objects like, for instance, supernovae (Weiler and Sramek 1988) and broad absorption-line (BAL) quasars (Weymann and Foltz 1983).

For extreme velocities $(v \geq 0.2 c)$, we naturally expect that the classical theory will cease to provide an accurate description of the observed line profiles. This can be easily understood by considering the Doppler effect which is essential in the formation of P Cygni line profiles (Beals 1929, 1931). Its relativistic expression is significantly more complex than in the low-velocity regime (see next section) and implies that the absolute frequency shift of photons scattered by material receding with a velocity $v$ (redshift) is smaller than the shift produced by material moving toward the observer at $-v$ (blueshift). The red emission wing of relativistic $\mathbf{P}$ Cygni line profiles will therefore be significantly narrower than the blue one, an asymmetry which is not present in the classical limit (see, e.g., the atlas of profiles due to Castor and Lamers 1979).

The aim of this paper is to derive the expression of line profiles formed in a relativistically expanding atmosphere assumed to be spherically symmetric and stationary. We

\footnotetext{
${ }^{1}$ Chercheur Qualifié au Fonds National de la Recherche Scientifique, Belgium.
}

restrict ourselves to the study of pure photon scattering by a two-level atom with complete redistribution in the fluid frame. In addition, we adopt the Sobolev approximation (Sobolev $1958,1960)$ for the treatment of the radiative transfer. If the very large velocities considered here are associated with similarly large velocity gradients throughout the envelope, this approximation is even more adequate than in cases describable in the classical limit. Our approach is quite different of that of Mihalas (1980) who uses the comoving-frame formalism to study the transfer of the continuum radiation in relativistic flows.

After briefly recalling the basic equations and notations ( II), we discuss in $\S$ III the modifications undergone by the surfaces of equal frequency when the fluid velocity becomes comparable to that of light. These surfaces are especially useful in order to visualize the transfer of line photons through the envelope. Following a probabilistic point of view, we derive in $\S$ IV the expression of transfer quantities such as the optical depth, the escape probabilities and the source function. This allows us to determine, in $\S \mathrm{V}$, the relativistic expression of a $\mathbf{P}$ Cygni line profile. In $\S$ VI, we study the first order moment of relativistic line profiles, a quantity which is particularly useful for the determination of mass-loss rates. Numerical applications are presented in $\S$ VII. Finally, conclusions form the last section.

Different formalisms based on the Sobolev approximation 
have been used to derive the classical expression of P Cygni line profiles (Castor 1970; Lucy 1971; Surdej 1979). In this paper, we follow the probabilistic approach developed by Surdej which, as we shall see, is quite easy to generalize to the relativistic case. We will frequently refer to Paper I (Surdej 1977) in which the classical escape probabilities and source function are derived and to Papers II and III (Surdej 1979, 1982) where line profiles are discussed.

\section{SOME BASIC EQUATIONS}

If we apply to the photon four-momentum the Lorentz transformation between a rest frame (observer's frame) and a frame moving at a constant velocity $v$ (comoving or fluid frame), it is easy to derive the well-known relations

$$
\begin{gathered}
v_{0}=v \gamma(1-\mu \beta), \\
\mu_{0}=\frac{\mu-\beta}{1-\mu \beta},
\end{gathered}
$$

where

$$
\begin{gathered}
\gamma=\frac{1}{\sqrt{1-\beta^{2}}}, \\
\beta=\frac{v}{c},
\end{gathered}
$$

$\mu=\cos \theta$ being the angle-cosine describing the direction of the photon propagation and $v$ the frequency (Mihalas 1978). Both $\mu$ and $v$ are measured in the rest frame while quantities with the suffix zero are measured in the comoving frame. As usual, $c$ represents the light velocity. We immediately see from equation (1) - the relativistic form of the Doppler effect-that the absolute frequency shift $\left|v_{0}-v\right|$ depends on the sign of $\mu$ for velocities comparable to that of light. For $\mu=0$, this shift is no longer equal to zero.

Let us now consider the transformations for the opacity $\left(\alpha_{v}\right)$, the emissivity $\left(\epsilon_{v}\right)$ and the specific intensity $\left(I_{v}\right)$. As shown by Thomas (1930), Mihalas (1978), and Mihalas and Mihalas (1984), the requirement that independent observers in both rest and comoving frames measure the same number of photon events leads to the following relations:

$$
\begin{gathered}
\frac{I_{v}}{v^{3}}=\frac{I_{v_{0}}^{0}}{v_{0}^{3}}, \\
v \alpha_{v}=v_{0} \alpha_{v_{0}}^{0}, \\
\frac{\epsilon_{v}}{v^{2}}=\frac{\epsilon_{v_{0}}^{0}}{v_{0}^{2}},
\end{gathered}
$$

where, again, all quantities with the suffix zero are measured in the comoving frame. We also have the useful relations

$$
\begin{gathered}
v^{2} d \omega=v_{0}^{2} d \omega_{0}, \\
v d v d \omega=v_{0} d v_{0} d \omega_{0},
\end{gathered}
$$

$d \omega$ representing a solid angle element measured in the rest frame.

\section{THE SURFACES OF EQUAL FREQUENCY}

Let us consider a spherically symmetric atmosphere expanding around a central core of radius $r_{c}$. The velocity of the flow $v(r)-r$ denoting the radial distance to the core-is supposed to increase continuously from $v_{c}$, the velocity at the surface of the core, to $v_{\infty}$, the terminal velocity.
If we assume that the fluid is populated by two-level $(1 \rightleftharpoons 2)$ atoms emitting photons at the comoving line center frequency $v_{12}^{0}$, all photons seen by a rest-observer with a specified frequency $v$ are emitted from surfaces satisfying the relation (see eq. [1])

$$
v_{12}^{0}=v \gamma(r)[1-\mu \beta(r)] \text {. }
$$

Solving this equation in $r$ and $\mu$ for different values of $v$ provides us with the surfaces of equal frequency $v$. Strictly speaking, these surfaces should be considered as thin shells because the thermal velocities are not exactly equal to zero when compared to the macroscopic velocity field. Now, if we define the dimensionless frequency

$$
X=-\frac{v-v_{12}^{0}}{v_{\max }-v_{12}^{0}},
$$

$v_{\max }$ representing the maximum frequency which corresponds to the terminal velocity $+v_{\infty}$, we easily find that

$$
X=-\frac{\left[\sqrt{1-\beta^{2}} /(1-\mu \beta)\right]-1}{\sqrt{\left(1+\beta_{\infty}\right) /\left(1-\beta_{\infty}\right)}-1},
$$

where $\beta_{\infty}$ is equal to $v_{\infty} / c$. So defined, the dimensionless frequency $X$ always satisfies the relation

$$
-1 \leq X \leq \sqrt{\frac{1-\beta_{\infty}}{1+\beta_{\infty}}}=X_{\infty}^{R},
$$

from which we immediately notice that no photons of frequencies $X \geq X_{\infty}^{R}$ are emitted toward the observer.

Adopting the velocity law $v(r)=v_{c}+\left(v_{\infty}-v_{c}\right)\left(1-r_{c} / r\right)$, representative of outward accelerating envelopes (see $\S$ VII), we have computed surfaces of equal frequency $X$ for different values of $\beta_{\infty}$. Examples are illustrated in Figures $1 a-1 d$. We immediately see that, unlike in the classical case, the surfaces strongly depend on the value of the terminal velocity and that very unsymmetric parts of the atmosphere may contribute to the frequencies $X$ and $-X$.

It is also interesting to consider the surfaces of equal frequency obtained for velocity laws of the type $v(r) \propto r^{n}$. Such surfaces are illustrated in Figures $2 a-2 d$ and show similar deformations for increasing values of $\beta_{\infty}$. But in addition, at very high velocities, we see that photons emitted toward the observer may intercept the same surface a second time and consequently be reabsorbed. This corresponds to the appearance of distant interactions in highly relativistic flows, a situation already known for the case of decelerated or nonmonotonic flows (see Rybicki and Hummer 1978 and Paper II). It is clear that such interactions completely modify the transfer of line radiation and may be of fundamental importance when evaluating the amplitude of the radiative forces. We should nevertheless note that these interactions are only present for very high values of $\beta$ and never for velocity distributions of the type considered in $\S$ VII. We will therefore not discuss in more detail this effect in the remainder of this paper.

We emphasize that the strong and nonlinear dependence of the surfaces of equal frequency upon $\beta_{\infty}$ forces us to consider the terminal velocity as a totally independent parameter when computing line profiles.

\section{PHOTON ESCAPE PROBABILITY AND SOURCE FUNCTION}

For the treatment of line transfer, we follow the probabilistic approach (see Paper I). This approach is particularly inter- 

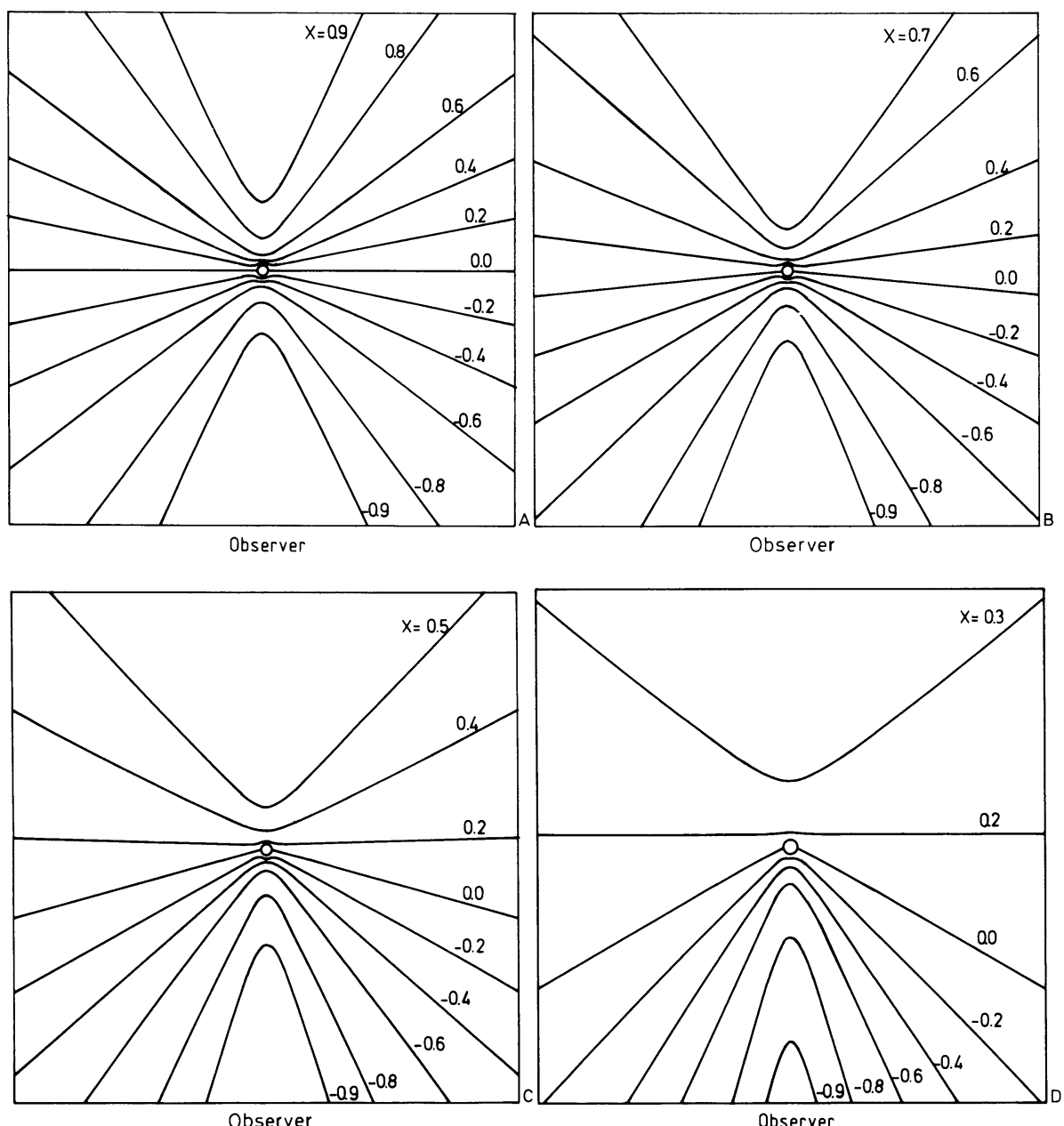

Fig. 1. - $(a-d)$ Evolution of the surfaces of equal frequency $X$ as a function of $\beta_{\infty}=0.01$ ( $a$; see the classical case), (b) $0.2,(c) 0.5$, and $(d) 0.8$ for a typical velocity law ( $B$ of eq. [72]). The surfaces actually extend to infinity, although for presentation they have been limited by boxes of $80 \times 80$ in units of $r_{c}$. Let us also recall that no photons are emitted at frequencies $X>X_{\infty}^{R}$ where $X_{\infty}^{R}=(a) 1.0,(b) 0.82,(c) 0.58$, and $(d) 0.33$.

esting in the relativistic case because we can easily manipulate invariants; a probability being nothing else than a ratio of photon numbers that is identical in both the rest and the comoving frames.

Since photon number must be carefully distinguished from energy, we prefer to use hereafter quantities like $I_{v}^{N}=I_{v} / h v$ and $\epsilon_{v}^{N}=\epsilon_{v} / h v, h$ denoting the Planck constant. The transformation laws of such quantities are immediately derived from equations $(5)-(7)$.

In order to define the useful transfer quantities (optical depth, absorption, or escape probabilities, etc.), we set ourselves in the rest frame where it is easier to follow the trajectories of atoms as well as photons. While most of these quantities are frame independent, it is often more convenient to calculate them in the comoving frame where, for example, the opacity and the emissivity are isotropic. Because the flow is accelerated, we must consider transformations between the rest frame and a series of frames which move at constant velocity, coinciding instantaneously with the fluid at a selected point.

In addition to the Sobolev approximation which states that most of the physical characteristics of the fluid can be considered as constant at some-small-distance scale (see the $\S \mathrm{IV} a$ ), we assume that the material advection is negligible. Because this latter approximation is certainly not valid for ultrarelativistic flows $(\beta \simeq 1)$-due to the time dilatation the distance traveled by a fluid element during the lifetime of an excited atomic state may become comparable to the Sobolev distance scale-we restrict our discussion to reasonable relativistic velocities (typically $\beta_{\infty} \leq 0.8$ ) which, in any case, encompass most of the existing observations.

\section{a) Relativistic Expression of the Photon Escape Probability}

Let us follow a photon emitted at a point $s_{E}$ in the moving fluid and seen, by a rest observer, at a frequency $v$ and propagating along a direction with abcissae $s$. This situation is illustrated schematically in Figure 3. This photon has a probability $d \tau_{v}=\alpha_{v}(s) d s$ of being absorbed between $s$ and $s+d s$ and therefore a probability $e^{-\tau_{v}}$ of leaving the envelope without any absorption, the optical depth $\tau_{v}$ taking the form

$$
\tau_{v}=\int_{S_{E}}^{\infty} \alpha_{v} d s .
$$

In order to easily calculate $\alpha_{v}$, we consider comoving frames coinciding instantaneously with the fluid at each point $s$ along the trajectory. In these frames, the opacity can be expressed by

$$
\alpha_{v_{0}}^{0}(s)=\alpha_{12}^{0}(r) \Phi_{0}\left(v_{0}-v_{12}^{0}\right)
$$



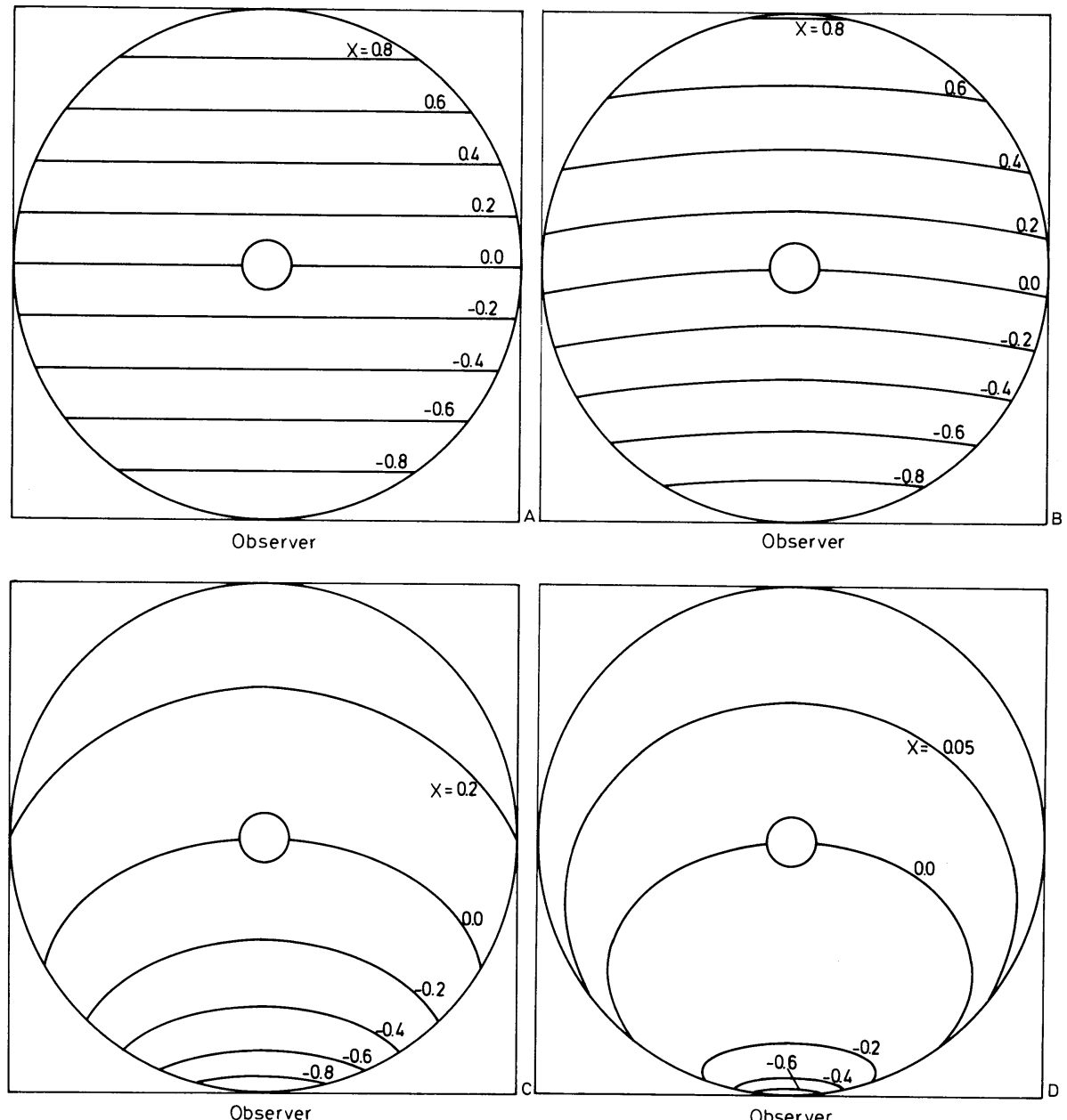

Fig. 2. $-(a-d)$ As in Fig. 1a-d, we see the evolution of the surfaces of equal frequency $X$ as a function of $\beta_{\infty}=(a) 0.01,(b) 0.2,(c) 0.8$, and $(d) 0.98$ but for the velocity law $v(r) / v_{\infty}=r / r_{\max }$ where $r_{\max }$, the maximum radial dimension of the envelope, is taken equal to $10 r_{c}$. The values of $X_{\infty}^{R}$ are, respectively, (a) $1.0,(b) 0.82,(c)$ 0.58 , and $(d) 0.10$. The appearance of distant radiative interactions in the moving envelope are clearly visible on $(d)$.

where $\alpha_{12}^{0}(r)$ is the total opacity in the transition $1 \rightleftharpoons 2$ and $\Phi_{0}\left(v_{0}-v_{12}^{0}\right)$ the atomic absorption profile. This absorption profile is assumed to be zero outside of the frequency interval

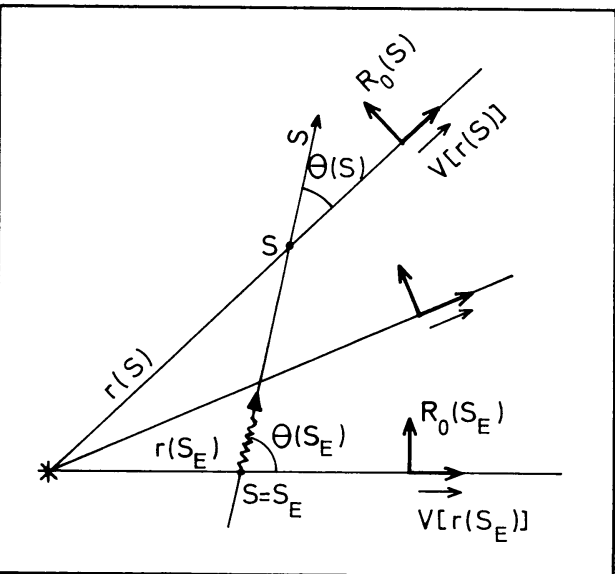

Fig. 3.-For a rest observer, a photon emitted at the point $s_{E}$ is seen to travel along a direction with abcissae $s$. At each point of the trajectory, we can associate a reference frame moving with a constant velocity $v[r(s)]$ and coinciding instantaneously with the fluid at this point.
$\left[v_{0}-\Delta v_{0} / 2, v_{0}+\Delta v_{0} / 2\right] ;$ the width $\Delta v_{0}$ includes the Doppler line broadening caused by thermal agitation, microturbulence, etc. As usual, this profile is normalized by the relation

$$
\int \Phi_{0}\left(v_{0}-v_{12}^{0}\right) d v_{0}=1 \text {. }
$$

The rest observer can transform expression (15) with the help of relation (6) and write the optical depth

$$
\tau_{v}=\int_{S_{E}}^{\infty} \frac{v_{0}(s)}{v} \alpha_{12}^{0}(s) \Phi_{0}\left(v_{0}-v_{12}^{0}\right) d s .
$$

Let us now estimate the probability that if a photon is emitted at the point $s_{E}$ (see Fig. 3), it is emitted in the frequency interval $[v, v+d v]$ and in the solid angle interval $[\omega, \omega+d \omega]$. This probability, while frame independent, is more conveniently calculated in the comoving frame where we have assumed complete redistribution in frequency and in direction. It takes the simple form

$$
\Phi_{0}\left(v_{0}^{E}-v_{12}^{0}\right) d v_{0}^{E} \frac{d \omega_{0}}{4 \pi}
$$

where

$$
v_{0}^{E}=v \gamma\left(s_{E}\right)\left[1-\mu\left(s_{E}\right) \beta\left(s_{E}\right)\right],
$$


$d \omega_{0}$ being the comoving solid angle corresponding to $d \omega$. The mean probability that a photon emitted at $s_{E}$ directly escapes the atmosphere may therefore be written

$$
\beta_{12}^{1}(r)=\int_{\Omega_{0}=4 \pi} \int_{-\infty}^{+\infty} e^{-\tau_{v}} \Phi_{0}\left(v_{0}^{E}-v_{12}^{0}\right) d v_{0}^{E} \frac{d \omega_{0}}{4 \pi} .
$$

In a very rapidly accelerating fluid, a photon of given frequency can only be absorbed within a small region of the envelope whose thickness $\Delta S(r, \mu)$ is directly related to the absorption profile width $\Delta v_{0}$ via the Doppler relation. The approximation introduced by Sobolev $(1958,1960)$ consists in assuming that both the physical and the kinematic fluid properties, except for the fluid velocity, are constant along spatial distances $\Delta S$. It is adequate whenever the thickness $\Delta S$ is very small compared to the envelope dimensions, i.e., if the velocity gradient is high and if the absorption width $\Delta v_{0}$ is small enough. Using this approximation after changing in equation (17) the variable of integration from $s$ to $v_{0}$, the expression of the escape probability $\beta_{12}^{1}$ reduces to

$$
\beta_{12}^{1}=\int_{\Omega_{0}=4 \pi} \frac{1-e^{-\tau_{12}}}{\tau_{12}} \frac{d \omega_{0}}{4 \pi}
$$

and finally, with equation (8), to

$$
\beta_{12}^{1}=\int_{-1}^{+1} \frac{1-e^{-\tau_{12}}}{\tau_{12}} \frac{d \mu}{2 \eta^{2}}
$$

where

$$
\begin{gathered}
\tau_{12}=\frac{\alpha_{12}^{0}}{v_{12}^{0}} \eta^{2}\left|\left(\frac{\partial \eta}{\partial s}\right)^{-1}\right| \\
\eta(r, \mu)=\gamma(1-\mu \beta) .
\end{gathered}
$$

Differentiating equation (24), we easily obtain

$$
\begin{aligned}
& \tau_{12}(r, \mu)=\tau_{12}^{r} \\
& \times\left|\frac{(1-\mu \beta)^{2}}{(1-\beta)\left[\mu(\mu-\beta)+\left(1-\mu^{2}\right)\left(1-\beta^{2}\right)(d \ln r / d \ln \beta)\right]}\right|, \\
& \tau_{12}^{r}(r)=\tau_{12}(\mu=+1)=\frac{\alpha_{12}^{0}}{v_{12}}\left(\frac{d \beta}{d r}\right)^{-1} \frac{(1-\beta)}{\gamma} .
\end{aligned}
$$

The $\beta_{12}^{1}$ and $\tau_{12}$ expressions are more complex than in the classical case (see Paper I). In particular, they are no longer symmetric in $\mu$. This is illustrated in Figure 4 where we can note that for relativistic velocities, the photon escape probability $\left(1-e^{-\tau_{12}}\right) / \tau_{12}$ is much greater in the direction of motion $(\mu=+1)$ than in the opposite one $(\mu=-1)$. Nevertheless, the Sobolev optical depth $\tau_{12}$ can still be written with the general and meaningful expression

$$
\tau_{12}=\frac{\alpha_{12}^{0}}{\Delta v} \Delta S
$$

$\Delta v$ being the rest frequency interval corresponding to $\Delta v_{0}$. It is important to recall that in the framework of the Sobolev approximation, a photon of given frequency can only be scattered within a very small region of the envelope before finally escaping it. The line transfer may therefore be considered as purely local and the Sobolev optical depth $\tau_{12}$ is sufficient to characterize the radiation-matter interactions at each point in the envelope.

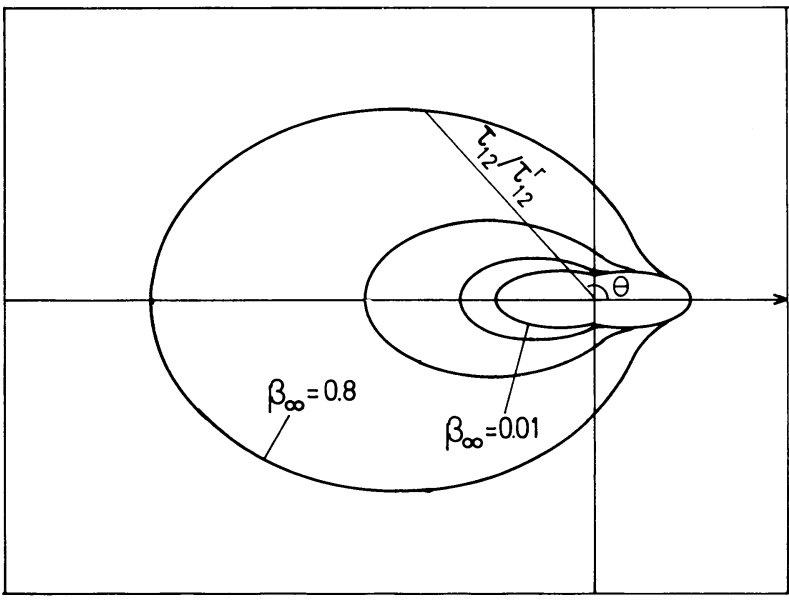

FIG. 4.-The ratio $\tau_{12}(r, \mu) / \tau_{12}^{r}(r)$ is represented here in a polar diagram for different values of $\beta_{\infty}(0.01,0.2,0.5$, and 0.8$)$. It is calculated at the distance $r=5 r_{c}$ from the central core for a typical velocity law ( $B$ of eq. [72]). The direction of motion is indicated. For $\mu=\cos \theta=1$, we have always $\tau_{12} / \tau_{12}^{r}=$ 1 while in the opposite direction $(\mu=-1)$, this ratio depends strongly on the value of $\beta_{\infty}$.

\section{b) Relativistic Expression of the Source Function}

To derive the expression of the source function we first need to evaluate the mean intensity of the radiation field. This is more easily done in the comoving frame where the process of emission is isotropic. In this frame, we denote by $J_{12}^{N, 0}$ the mean specific intensity weighted on the line profile and divided by $h v_{12}^{0}$.

${ }_{J_{12}^{N, 0}}^{12}$ contains two major contributions: the first one is due to photons issued from the central core (the distant contribution) and the other one to photons locally scattered (the local contribution). Let us first estimate the distant contribution assuming that the core is emitting a continuum whose intensity $I_{c}^{N}$, measured by the rest observer, is constant over the line profile and does not show any limb darkening effect. For this observer, the intensity of the radiation emitted at a frequency $v$ by a point of the core surface and reaching another point located in the envelope, is equal to $I_{c}^{N} e^{-\tau_{v}}, \tau_{v}$ representing the optical depth between the core surface and the considered point in the envelope. Measured in the comoving frame which coincides instantaneously with the fluid at this point, this intensity is equal to $\eta^{2} I_{c}^{N} e^{-\tau_{v}}$, following equation (5) and remembering that $I_{c}^{N}$ has also been divided by $h v$. Averaging this quantity in the comoving frame over both the line absorption profile and the directions, we obtain the distant contribution to the mean intensity whose expression

$$
\int_{\Omega_{0}=4 \pi} \int_{-\infty}^{+\infty} \eta^{2} I_{c}^{N} e^{-\tau_{v}} \Phi_{0}\left(v_{0}-v_{12}^{0}\right) d v_{0} \frac{d \omega_{0}}{4 \pi}
$$

reduces, in the framework of the Sobolev approximation, to

$$
I_{c}^{N} \int_{4 \pi W} \frac{1-e^{\tau_{12}}}{\tau_{12}} \frac{d \omega}{4 \pi},
$$

$W$ denoting the geometrical dilution factor

$$
\left[1-\sqrt{1-\left(r_{c} / r\right)^{2}}\right] / 2 .
$$

The quantity

$$
\beta_{12}^{3}(r)=\int_{4 \pi W} \frac{1-e^{-\tau_{12}}}{\tau_{12}} \frac{d \omega}{4 \pi}=\int_{1-2 W}^{1} \frac{1-e^{-\tau_{12}}}{\tau_{12}} \frac{d \mu}{2}
$$


represents the mean penetration probability of photons emitted from the central core. It is important to note that in a relativistically expanding envelope, $\beta_{12}^{3}$ is no longer equal to the mean probability that a photon escapes directly the envelope and intercepts the central core. This latter probability is in fact equal to (see eq. [22])

$$
\int_{-1}^{-(1-2 W)} \frac{1-e^{-\tau_{12}}}{\tau_{12}} \frac{d \mu}{2 \eta^{2}}
$$

The difference between escape and penetration mean probabilities is simply due to different assumptions about the directional variation of the radiation field: for $\beta_{12}^{1}$, we assume the isotropic emission in the local comoving frame and for $\beta_{12}^{3}$, the isotropic emission from the distant core in the rest frame. Asides from the factor that incorporates the direction behavior of the radiation field, the penetration and escape probabilities for one direction are the same: $\left(1-e^{-\tau_{12}}\right) / \tau_{12}$.

The local contribution to the mean intensity $J_{12}^{N, 0}$ can be evaluated following a similar reasoning (see also Paper I). However since the Sobolev line transfer is purely local, the photon input-output being controlled at each point in the envelope by the probabilities $\beta_{12}^{3}$ and $\beta_{12}^{1}$, it is clear that in the comoving frame this local contribution takes the same form as in the classical approximation, i.e., $S_{12}^{N, 0}\left(1-\beta_{12}^{1}\right)$ which simply represents the fraction of photons having not directly escaped the envelope, $S_{12}^{N, 0}(r)=\epsilon_{12}^{N, 0} / \alpha_{12}^{0}$ denoting the source function measured in the comoving frame. Combining these two contributions, we may write

$$
J_{12}^{N, 0}=S_{12}^{N, 0}\left(1-\beta_{12}^{1}\right)+\beta_{12}^{3} I_{c}^{N} .
$$

Because we only consider the pure scattering of line photons, we have also

and consequently

$$
J_{12}^{N, 0}=S_{12}^{N, 0}
$$

$$
S_{12}^{N, 0}=\frac{\beta_{12}^{3}}{\beta_{12}^{1}} I_{c}^{N}
$$

As we shall see in $\S$ VII, the numerical values of $S_{12}^{N, 0}$ only slightly depend $v_{\infty}$, even if $v_{\infty}$ is relativistic. But, measured in the rest frame, the source function $S_{12}^{N}(r, \mu)=S_{12}^{N, 0} / \eta^{2}$ is clearly anisotropic for relativistic velocities, taking higher values along the direction of motion.

\section{RELATIVISTIC EXPRESSION OF THE LINE PROFILE FUNCTION}

In deriving the expression of the line profile function $E(X) / E_{c}$, we first assume - as in Paper II-that the central core is pointlike. This constitutes a reasonable approximation for the case of accelerated flows, and it allows us to follow step by step a photon emitted from the core with a better understanding of the basic line formation mechanism as well as of the relativistic effects. The expression of the line profile is subsequently, and quite easily, generalized to the case of a central core with finite dimensions.

\section{a) The Pointlike Core Approximation}

Let us consider a photon emitted by a pointlike core along a radial direction $(\mu=+1)$ and with a dimensionless frequency $X^{\prime}$. In the framework of the Sobolev approximation, this photon can only interact with the material at a given point $r\left(X^{\prime}\right)$ in the envelope, fixed by the Doppler relation and the velocity distribution $\beta(r)$ characterizing the flow. We therefore only consider photons with frequencies $X^{\prime}$ in the interval [ -1 , $\left.X_{\min }\right]$ corresponding to the distance interval $\left[+\infty, r_{c}\right]$, the value of $X_{\min }$ being found from equation (12) where we replace $\beta$ by $\beta_{c}=v_{c} / c$ and $\mu$ by +1 . If absorbed at $r\left(X^{\prime}\right)$, this photon of initial frequency $X^{\prime}$ can escape the envelope along any direction with $\mu$ in the interval $[-1,+1]$, i.e., with a frequency $X$ such that $X(\mu=+1) \leq X \leq X(\mu=-1)$ or

$$
X^{\prime} \leq X \leq-\sqrt{\frac{1-\beta}{1+\beta}} X^{\prime}=X^{R} .
$$

This frequency interval $\left[X^{\prime}, X^{R}\right]$ may be up to twice narrower than in the classical case where it is equal to $\left[X^{\prime},-X^{\prime}\right]$. Let us note that the variable set $\left(X^{\prime}, X\right)$ is completely equivalent to the set $(r, \mu)$ and it will be advantageously used in the remainder of this paper.

The probability that this photon of frequency $X^{\prime}$ could be absorbed at the distance $r\left(X^{\prime}\right)$ is simply equal to

$$
P_{a}\left(X^{\prime}\right)=1-e^{-\tau_{12}^{\prime}},
$$

$\tau_{12}^{r}\left(X^{\prime}\right)$ denoting the radial optical depth defined by equation (26). If absorbed, the photon has a probability

$$
P_{n}\left(X^{\prime}\right)=\left(1-\beta_{12}^{1}\right)^{n}
$$

of undergoing $n$ other local scatterings. It will finally escape the envelope along directions toward the observer, i.e., within the interval $[\mu, \mu+d \mu]$ or similarly in the frequency interval $[X, X+d X]$, with a probability $P_{e}\left(X^{\prime}, X\right) d X$. Because we have assumed complete redistribution in the comoving frame, this latter probability is simply expressed by

$$
P_{e}\left(X^{\prime}, X\right) d X=\frac{1-e^{\tau_{12}}}{\tau_{12}} \frac{d X}{X^{R}-X^{\prime}},
$$

$\tau_{12}\left(X^{\prime}, X\right)$ denoting the Sobolev optical depth along the observer direction. The factor $d X /\left(X^{R}-X^{\prime}\right)$, equal to $d \mu_{0} / 2=$ $d \mu / 2 \eta^{2}$, indicates that photons are uniformly reemitted in the frequency interval $\left[X^{\prime}, X^{R}\right]$. Because this interval is narrower than in the classical case, the probability $d X /\left(X^{R}-X^{\prime}\right)$ that a photon scattered at $r\left(X^{\prime}\right)$ is reemitted with a frequency between $X$ and $X+d X$ may be significantly increased. Combining these different probabilities, we derive the probability that a photon emitted by the core at a frequency $X^{\prime}$ escapes the envelope toward the observer with a frequency in the interval $[X, X+d X]$ and after an arbitrary number of local scatterings:

$$
P\left(X^{\prime}, X\right) d X=P_{a}\left(X^{\prime}\right)\left[1+\sum_{n=1}^{\infty} P_{n}\left(X^{\prime}\right)\right] P_{e}\left(X^{\prime}, X\right) d X
$$

which reduces to

$$
P\left(X^{\prime}, X\right)=\frac{1-e^{-\tau_{12}^{r}}}{X^{R}-X^{\prime}} \frac{1-e^{-\tau_{12}}}{\beta_{12}^{1} \tau_{12}}
$$

Summing over all frequencies $X^{\prime}$ contributing to the observed frequency $X$, we immediately obtain the contribution to the line profile due to the scattered line photons,

$$
\frac{E^{S}(X)}{E_{c}}=\int_{X_{\mathrm{inf}}^{\prime}}^{X_{\text {sup }}^{\prime}} P\left(X^{\prime}, X\right) d X^{\prime},
$$

assuming that the central core emits photons in constant number over the frequency interval of interest. In absence of limb darkening, $E_{c}$ is equal to $\pi I_{c}^{N}$. The frequency interval 
$\left[X_{\text {inf }}^{\prime}, X_{\text {sup }}^{\prime}\right]$ may be found from relations (12), (13), and (35): photons reaching the observer with a frequency $X$ can only originate, if $X \leq 0$, from scattering of photons with an initial frequency $X^{\prime}$ in the interval $\left[-1, \min \left(X, X_{\min }\right)\right]$ and, if $X>0$, I from the scattering of photons with a frequency $X^{\prime}$ in the interval $\left[-1, \min \left(X_{c}^{\prime}, X_{\min }\right)\right]$, where $X_{c}^{\prime}$ is solution of $\mu\left(X_{c}^{\prime}, X\right)=-1$, i.e., $X_{c}^{\prime}=-X /\left(1+X-X / X_{\infty}^{R}\right)$. Let us recall that for frequencies $X>X_{\infty}^{R}$ there are no scattered photons $\left[E^{S}(X) / E_{c}=0\right]$ so that the scattered contribution becomes very asymmetrical for increasing values of $\beta_{\infty}$.

Finally, the total line profile function $E(X) / E_{c}$ is obtained by adding the contribution $E^{S}(X) / E_{c}$ due to the scattered line photons and the contribution $E^{A}(X) / E_{c}$ due to photons reaching directly the observer, this latter contribution being expressed by

$$
\begin{array}{ll}
\frac{E^{A}(X)}{E_{c}}=1-P_{a}(X) & \text { if } X \leq X_{\min }, \\
\frac{E^{A}(X)}{E_{c}}=1 & \text { if } X>X_{\min } .
\end{array}
$$

\section{b) The Central Core with Finite Dimensions}

For a central core having finite dimensions, one must consider that back-scattered photons may strike it and that these cannot reach the observer any longer. This is the well-known occultation effect. In addition, photons originating from the core are not only emitted along radial directions $(\mu=+1)$ but also along inclined ones. We call this the inclination effect (see Paper III).

Considering first the contribution $E^{S}(X) / E_{c}$ due to scattered photons, we take into account the occultation effect by replacing, for positive $X$ values, the frequency limit $X_{\text {sup }}^{\prime}$ by $X_{\text {sup }}^{\prime \prime}=$ $\min \left(X_{c}^{\prime \prime}, X_{\min }\right)$ where $X_{c}^{\prime \prime}$ is solution of $\mu_{c}\left(X_{c}^{\prime \prime}, X\right) \stackrel{\text { sup }}{=}-1$ $+2 W\left[r\left(X_{c}^{\prime \prime}\right)\right]$. It is also clear that in equation (39), the factor $P_{a}\left(X^{\prime}\right)$ must be replaced by a new quantity $P_{a}^{\prime}\left(X^{\prime}\right)$ which accounts for the fraction of photons emitted by the core along nonradial directions. Recalling that $P_{a}^{\prime}\left(X^{\prime}\right) I_{c}^{N}$ and $\beta_{12}^{3} I_{c}^{N}$ are, respectively, proportional to the number of core photons absorbed at $r\left(X^{\prime}\right)$ and to the number of core photons arriving at $r\left(X^{\prime}\right)$, we have the relation

$$
\frac{P_{a}^{\prime}\left(X^{\prime}\right)}{P_{a}\left(X^{\prime}\right)}=\frac{\beta_{12}^{3}\left[r\left(X^{\prime}\right)\right]}{\beta_{12}^{3}\left(r / r_{c} \rightarrow \infty\right)}
$$

which reduces to

$$
P_{a}^{\prime}\left(X^{\prime}\right)=\beta_{12}^{3}\left(X^{\prime}\right) \tau_{12}^{r}\left(X^{\prime}\right) 4 L^{2}\left(X^{\prime}\right),
$$

where $L=r / r_{c}$. The contribution due to scattered photons can therefore be written

$$
\frac{E^{S}(X)}{E_{c}}=\int_{X_{\text {inf }}^{\prime}}^{X_{\text {sup }}^{\prime \prime}} \frac{\beta_{12}^{3}}{\beta_{12}^{1}} \frac{1-e^{-\tau_{12}}}{\tau_{12}} \frac{4 L^{2} \tau_{12}^{r}}{X^{R}-X^{\prime}} d X^{\prime} .
$$

By integrating over the core surface, we easily show (see Paper III) that the contribution $E^{A}(X) / E_{c}$ due to photons reaching the observer without having suffered any scattering may be generalized to

$$
\begin{array}{ll}
\frac{E^{A}(X)}{E_{c}}=2 \int_{0}^{1} e^{-\tau_{12}\left(X^{\prime}, \mu\right)} \mu_{\star} d \mu_{\star} & \text { if } X<X_{\star}, \\
\frac{E^{A}(X)}{E_{c}}=1 & \text { if } X \geq X_{\star},
\end{array}
$$

where $X^{\prime}$ is solution of $1-\left[1-\mu^{2}\left(X^{\prime}, X\right)\right] L^{2}\left(X^{\prime}\right)=\mu_{\star}^{2}$. In this relation, the value of $\tau_{12}$ must be set to zero if no solution of $X^{\prime} \leq X_{\min }$ can be found. The frequency $X_{\star}$, solution of $\mu\left(X_{\min }, X_{\star}\right)=0$, is explicitly expressed by

$$
X_{\star}=\frac{B_{\infty} X_{\min }^{2}}{\left[\left(1-B_{\infty} X_{\min }\right)^{2}+1\right]} \geq 0
$$

where, for simplicity, we have introduced

$$
B_{\infty}=\frac{v_{\max }-v_{12}^{0}}{v_{12}^{0}}=\sqrt{\frac{1+\beta_{\infty}}{1-\beta_{\infty}}}-1 .
$$

It is interesting to note that photons with positive $X$ frequencies can be absorbed in the envelope. This relativistic effect $\left(X_{\star}=0\right.$ in the classical limit) remains small and needs high $\left|X_{\min }\right|$ values in order to become significant. This effect is implicitly included in the expression of the emission profile (eq. [45]).

\section{THE FIRST-ORDER MOMENT OF UNSATURATED RELATIVISTIC P CYGNI LINE PROFILES}

The first-order moment of a P Cygni line profile has been introduced by Castor, Lutz, and Seaton (1981) as a powerful tool for deriving mass-loss rates. Subsequent work by Surdej $(1982,1983 b)$ has shown that a unique linearity relation does actually exist between the first-order moment of an unsaturated line profile and the fractional mass-loss rate, irrespective of various physical (opacity distribution, collisions, limb darkening, etc.) and geometrical (velocity law, rotation, etc.) conditions prevailing in the expanding envelope as well as of any Sobolev-type approximations used for the transfer of line radiation. In this section we investigate the behavior of the firstorder moment when the flow becomes relativistic.

The $n$th order moment of a line profile can be defined as

$$
W_{n}^{R}=\left(\frac{1}{v_{\max }-v_{12}^{0}}\right)^{n+1} \int_{-\infty}^{+\infty}\left(\frac{E(v)}{E_{c}}-1\right)\left(v_{12}^{0}-v\right)^{n} d v
$$

an expression which, in terms of the dimensionless frequency $X$, easily reduces to

$$
W_{n}^{R}=\int_{-1}^{X_{\infty}^{R}}\left[\frac{E(X)}{E_{c}}-1\right] X^{n} d X .
$$

In the low-velocity regime, this definition of $W_{n}^{R}$ is exactly equivalent to that of Castor, Lutz, and Seaton (1981).

Let us now evaluate the first-order moment of unsaturated line profiles considering first the pointlike core approximation. For such profiles, the first-order moment will be denoted by $W_{1}^{R, 0}$.

\section{a) The Pointlike Core Approximation}

The expression of the moment $W_{1}^{R, 0}$ can be easily obtained by combining equations (41), (42), and (50). We nevertheless prefer to follow a rather different approach which provides us with a better understanding of the moment properties.

Up to now, we have evaluated the line profile expression by summing at each frequency $X$ the different fractions of line photons reaching an observer after having undergone an arbitrary number of scatterings, i.e., we have considered the envelope as a juxtaposition of surfaces of equal frequency $X$. On the other hand, we can imagine the envelope as an assembly of shells of radius $X^{\prime}$ and thickness $\Delta X^{\prime}$. Because the 
Sobolev transfer is purely local, the line profile formed in the whole envelope is nothing else than the sum of the profiles due to such shells. The same applies for the moment $W_{1}^{R, 0}$ and we can write

$$
W_{1}^{R, 0}=\int_{-1}^{x_{\min }} W_{1}^{R, 0}\left(X^{\prime}\right) d X^{\prime},
$$

$W_{1}^{R, 0}\left(X^{\prime}\right) d X^{\prime}$ representing the first-order moment of the profile formed in the shell of radius $X^{\prime}$ and thickness $d X^{\prime}$. If the envelope is optically thin, the profile due to a shell is very simple (see Fig. 5a) and the corresponding moment $W_{1}^{R, 0}\left(X^{\prime}\right) d X^{\prime}$ can be evaluated by simply summing up elementary surfaces. From Figure $5 b$, we obtain

$$
\begin{aligned}
& W_{1}^{R, 0}\left(X^{\prime}\right) \Delta X^{\prime}=\tau_{12}^{r}\left|X^{\prime}\right| \Delta X^{\prime}-\frac{\tau_{12}^{r} \Delta X^{\prime}}{X^{R}-X^{\prime}}\left|X^{\prime}\right| \frac{\left|X^{\prime}\right|}{2} \\
&+\frac{\tau_{12}^{r} \Delta X^{\prime}}{X^{R}-X^{\prime}} X^{R} \frac{X^{R}}{2}
\end{aligned}
$$

or

$$
W_{1}^{R, 0}\left(X^{\prime}\right)=\tau_{12}^{r} \frac{X^{R}-X^{\prime}}{2}
$$

giving finally

$$
W_{1}^{R, 0}=\int_{-1}^{X_{\min }} \tau_{12}^{r}\left(X^{\prime}\right) \frac{\left(X^{R}-X^{\prime}\right)}{2} d X^{\prime} .
$$

It is interesting to note that the detailed expression of the line profile (eqs. [41] and [42]) is not necessary in order to calculate the moment $W_{1}^{R, 0}$ in the optically thin approximation. We also clearly see that the difference between this expression and the classical one (see Paper III) arises from the asymmetry of the relativistic emission profile.

Let us now develop the expression of the optical depth $\tau_{12}^{r}\left(X^{\prime}\right)$ given by equation (26). Neglecting the stimulated emission correction, the opacity $\alpha_{12}^{0}$ may be written

$$
\alpha_{12}^{0}=N_{1}^{0}\left(\frac{\pi e^{2}}{m c}\right)_{0} f_{12}^{0},
$$

or

$$
\alpha_{12}^{0}=n_{1} A(\mathrm{el}) N_{\mathrm{tot}}^{0}\left(\frac{\pi e^{2}}{m c}\right)_{0} f_{12}^{0},
$$

where $N_{1}^{0}$ represents the number of atoms in the lower state 1 measured per unit of proper volume, $f_{12}^{0}$ the oscillator strength, $A(\mathrm{el})$ the abundance of the element, $n_{1}$ the fractional abundance of the element's atoms which are in the lower state and $N_{\text {tot }}^{0}$ the total number of nuclei measured per unit of proper volume. The other quantities have their usual meaning. The suffix zero reminds us that the relevant quantities are defined in the comoving frame. If there is conservation of the total nucleus number, the equation of continuity leads to the relation

$$
4 \pi r^{2} v(r)\left(N_{\mathrm{tot}}^{0} \bar{\mu} M_{\mathrm{amu}}^{0}\right) \gamma=\dot{M},
$$

where $\dot{M}$ denotes the proper mass-loss rate, $\bar{\mu}$ the mean atomic weight of the nuclei and $M_{\mathrm{amu}}^{0}$ the unit of atomic mass. The Lorentz factor $\gamma$ appears in equation (57) because the volume of a fluid element $\left(\propto 1 / N_{\text {tot }}^{0}\right)$ is lower when measured in the observer frame. Combining these relations with the definitions of $X, X^{\prime}$, and $L$, we finally obtain

$$
\tau_{12}^{r}=K_{12}^{0} \dot{M} n_{1}\left(\frac{1}{c B_{\infty}}\right)^{2} \frac{2 d L}{L^{2}\left(X^{\prime}-X^{R}\right) d X^{\prime}}
$$

where

$$
K_{12}^{0}=\left(\frac{\pi e^{2}}{m c}\right)_{0} f_{12}^{0} \lambda_{12}^{0} \frac{A(\mathrm{el})}{4 \pi \bar{\mu} M_{\mathrm{amu}}^{0} r_{c}} .
$$

The moment $W_{1}^{R, 0}$ can therefore be written

$$
W_{1}^{R, 0}=K_{12}^{0} \frac{\dot{M}}{\left(c B_{\infty}\right)^{2}} \int_{1}^{\infty} n_{1} \frac{d L}{L^{2}},
$$

showing us that the linear relation between the mass-loss rate and the first-order moment of an unsaturated P Cygni line profile still holds in the relativistic case.

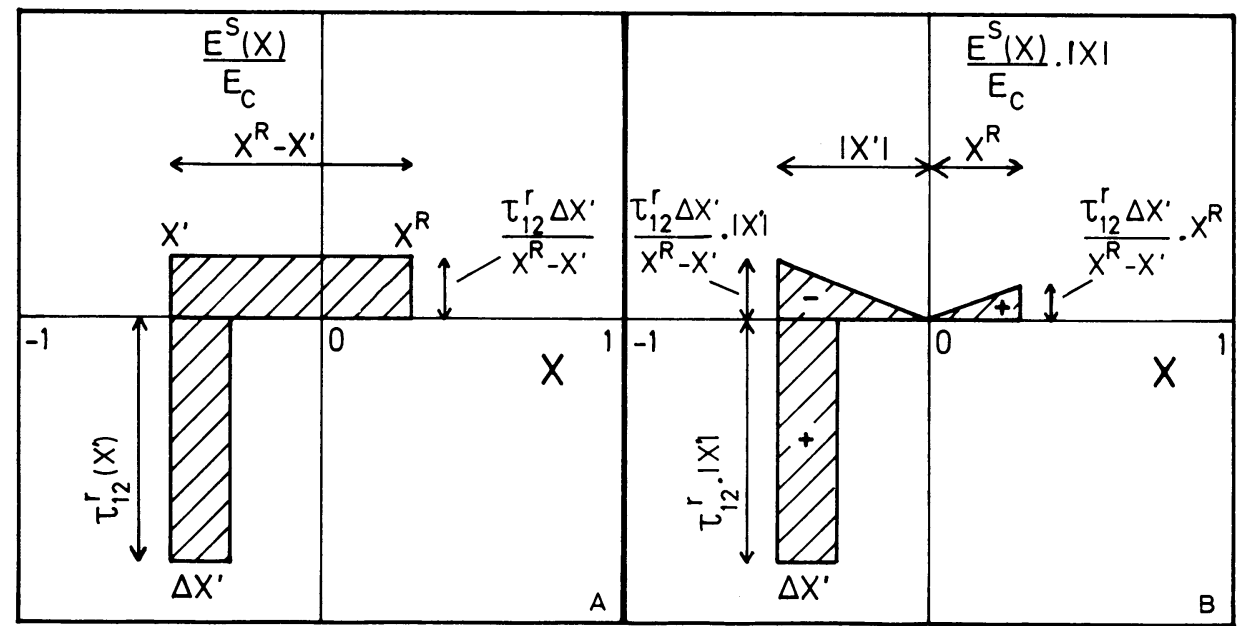

Fig. 5. - The first figure (a) illustrates schematically the very simple line profile issued from an optically thin shell of radius $X^{\prime}$ and thickness $\Delta X^{\prime}\left(\Delta X^{\prime}\right.$ is in reality much smaller than represented). Part of the photons emitted by the pointlike core are absorbed at the frequency $X^{\prime}$ and reemitted in the frequency interval [ $\left.X^{\prime}, X^{R}\right]$ (see, e.g., eq. [35]). The material being optically thin, the area of the absorption part of the line profile is equal to $\tau_{12}^{r}\left(X^{\prime}\right) \Delta X^{\prime}$. Because the photon number is conserved and because the core is assumed pointlike, $\tau_{12}^{r}\left(X^{\prime}\right) \Delta X^{\prime}$ also represents the area of the emission-line profile. Fig. $(b)$ represents schematically the line profile multiplied by $|X|$, as in the first-order moment definition (eq. [50]). This moment can be calculated by simply summing up the elementary surfaces (see text). 


\section{b) The Central Core with Finite Dimensions}

If we consider the finite dimensions of the central core, we can write the first-order moment as

$$
W_{1}^{R, 0}=\int_{-1}^{X_{\min }} \tau_{12}^{r} \frac{\left(X^{R}-X^{\prime}\right)}{2} \Omega\left(X^{\prime}\right) d X^{\prime},
$$

where the factor $\Omega\left(X^{\prime}\right)$ mainly accounts for the part of the scattered line photons which do not reach the observer due to occultation by the core. The expression of $\Omega\left(X^{\prime}\right)$ is fairly complex. It can be derived by replacing in the moment definition (50) the line profile by its expressions (45) and (46) where we impose $\tau_{12} \ll 1$. We find

$\Omega\left(X^{\prime}\right)=4 L^{2}\left(\frac{1-B_{\infty} X^{\prime}}{2-B_{\infty} X^{\prime}}\right)^{2}\left[W \frac{X_{c}^{2}}{X^{\prime 2}}-W-2 \frac{S_{1}\left(X^{\prime}\right)}{X^{\prime 2}}\right]$

where

$$
\begin{aligned}
S_{1}\left(X^{\prime}\right)=\frac{1}{B_{\infty}^{2}} & {\left[\ln \left(\frac{1-B_{\infty} X_{|c|}}{1-B_{\infty} X^{\prime}}\right)\right.} \\
& \left.\quad+\frac{1}{\left(1-B_{\infty} X_{|c|}\right)}-\frac{1}{\left(1-B_{\infty} X^{\prime}\right)}\right],
\end{aligned}
$$

with

$$
\begin{gathered}
X_{c}=\frac{1}{B_{\infty}}\left[1-\left(1-B_{\infty} X^{\prime}\right) \frac{1-\beta}{1+(1-2 W) \beta}\right], \\
X_{|c|}=\frac{1}{B_{\infty}}\left[1-\left(1-B_{\infty} X^{\prime}\right) \frac{1-\beta}{1-(1-2 W) \beta}\right] .
\end{gathered}
$$

Let us recall that in the classical limit the factor $\Omega\left(X^{\prime}\right)$ is simply equal to $1-W$ (see Paper III).

If we now replace $\tau_{12}^{r}$ by its expression (58) and define

$$
\begin{aligned}
\left\langle n_{1}\right\rangle & =\frac{\int_{1}^{\infty} n_{1} \Omega / L^{2} d L}{\int_{1}^{\infty} \Omega / L^{2} d L}, \\
q^{c} & =\int_{1}^{\infty} \frac{\Omega}{L^{2}} d L,
\end{aligned}
$$

we obtain the useful relation

$$
\dot{M}\left\langle n_{1}\right\rangle=W_{1}^{R, 0} \frac{\left(B_{\infty} c\right)^{2}}{q^{c} K_{12}^{0}} .
$$

\begin{tabular}{|c|c|c|c|}
\hline \multirow[b]{2}{*}{$\beta_{\infty}$} & \multicolumn{3}{|c|}{ VeLOCITY LAW } \\
\hline & $A$ & $B$ & $C$ \\
\hline $0.01 \ldots \ldots \ldots$ & 0.89 & 0.89 & 0.89 \\
\hline $0.20 \ldots \ldots \ldots$ & 0.89 & 0.89 & 0.89 \\
\hline $0.50 \ldots \ldots \ldots$ & 0.89 & 0.88 & 0.86 \\
\hline $0.80 \ldots \ldots \ldots$ & 0.88 & 0.86 & 0.81 \\
\hline
\end{tabular}

This general relation allows the immediate determination of mass-loss rates from the measurement of the moment $W_{1}^{R, 0}$ of unsaturated line profiles, if the average ionization fraction $\left\langle n_{1}\right\rangle$ is known. In principle, the normalization factor $q^{c}$ depends on both $\beta_{\infty}$ and the type of velocity law but, as we can see from Table 1 , this dependence is negligible for the considered

TABLE 1 models. Thus, as in the classical limit, equation (68) holds within a good approximation irrespective of the kind of velocity law chosen to represent the expanding atmosphere. In addition, the relative constancy of $q^{c}$ confirms the fact that the moment $W_{1}^{R, 0}$ constitutes a useful physical parameter.

If we now want to evaluate mass-loss rates from slightly or strongly saturated profiles we have to use $W_{1}^{R}-W_{1}^{R, 0}$ diagrams. Such diagrams are constructed numerically in the next section.

\section{NUMERICAL APPLICATIONS}

We present here a few examples of typical source functions, P Cygni line profiles and $W_{1}^{R}-W_{1}^{R, 0}$ diagrams computed when the velocities become comparable to that of light.

In all these applications, we use the first-order moment $W_{1}^{R, 0}$ to parameterize the opacity. From equations (58) and (68), the radial optical depth $\tau_{12}^{r}$ can be written

$$
\tau_{12}^{r}=W_{1}^{R, 0} \frac{n_{1}}{q^{c}\left\langle n_{1}\right\rangle} \frac{2 d L}{L^{2}\left(X^{\prime}-X^{R}\right) d X^{\prime}}
$$

or

$$
\tau_{12}^{r}=\frac{W_{1}^{R, 0}}{q^{c}\left\langle n_{1}\right\rangle} \tau^{r}\left(v / v_{\infty}\right)
$$

$\tau^{r}\left(v / v_{\infty}\right)$ representing the dependence of the opacity distribution as a function of $v / v_{\infty}$. The factor $\left\langle n_{1}\right\rangle$ is evaluated by making equations (61) and (70) consistent. In the remainder, we adopt the following opacity laws:

$$
\begin{array}{ll}
(\beta) & \tau^{r}\left(v / v_{\infty}\right)=1-v / v_{\infty}, \\
(\gamma) & \tau^{r}\left(v / v_{\infty}\right)=1, \\
(\delta) & \tau^{r}\left(v / v_{\infty}\right)=\left(1-v / v_{\infty}\right)^{1 / 2}, \\
(\epsilon) & \tau^{r}\left(v / v_{\infty}\right)=\left(1-v / v_{\infty}\right)^{2},
\end{array}
$$

which are of the form $\tau^{r}\left(v / v_{\infty}\right)=\left(1-v / v_{\infty}\right)^{\gamma}$. It is generally considered that this type of opacity laws provides a good description of the expanding atmospheres around early-type stars (Castor and Lamers 1979; Garmany et al. 1981). We also adopt the following velocity distributions:

$$
\begin{array}{ll}
(A) & v(L)=v_{c}+\left(v_{\infty}-v_{c}\right)(1-1 / \sqrt{L}), \\
(B) & v(L)=v_{c}+\left(v_{\infty}-v_{c}\right)(1-1 / L), \\
(C) & v(L)=v_{\infty} \sqrt{1-\left(1-v_{c}^{2} / v_{\infty}^{2}\right) / L},
\end{array}
$$

which are also thought to encompass most of the observed velocity fields prevailing in expanding envelopes (Castor et al. 1975; Castor and Lamers 1979; Olson 1981; Olson and Ebbets 1981). The various combinations of the different opacity and velocity laws lead to 12 distinct models. For consistency with our previous papers, we shall label them in the form $(A . \beta)$ for example, where $A$ denotes the type of velocity law and $\beta$ the type of opacity distribution. Finally, all our calculations have been done using four values of $\beta_{\infty}: 0.01,0.2,0.5$, and 0.8 , the first one corresponding to the classical limit. In all cases (except for Figs. $7 g-7 h$ ), we also take $\beta_{c} / \beta_{\infty}=0.01$.

The source function $S_{12}^{N, 0}$ is illustrated in Figures $6 a-b$ for two different values of the parameter $W_{1}^{R, 0}$. As we can see, the changes with $\beta_{\infty}$ remain reasonably small.

Figures $7 a-h$ illustrate typical $\mathbf{P}$ Cygni line profiles computed from equations (45) and (46). We immediately notice that the profiles now depend significantly on the value of the ter- 

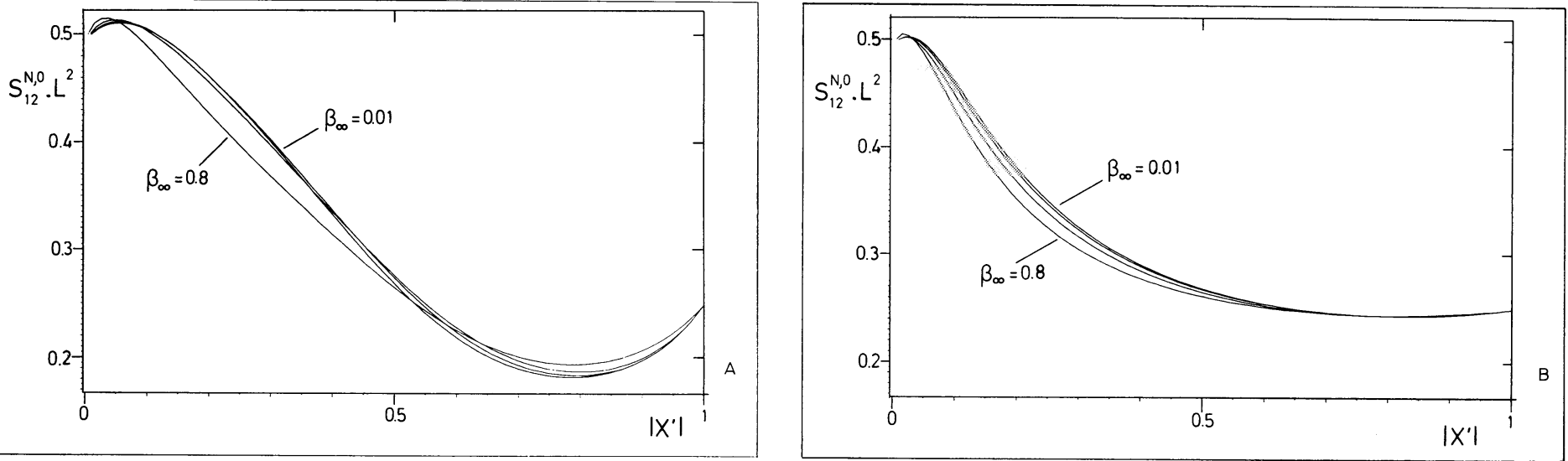

Fig. 6. - $(a-b)$ Examples of source functions $S_{12}^{N, 0}$ multiplied by $L^{2}$ to remove the main geometrical effect. They are computed for the $\beta_{\infty}$ values $0.01,0.2,0.5$, and 0.8 and for the typical model $(B . \beta)$. The parameter $W_{1}^{R, 0}$ has been taken equal to $(a) 1.0$ and $(b) 0.1$.

minal velocity (while they do not in the classical theory) but that rather high values of $\beta_{\infty}$ are nevertheless needed in order to cause severe modifications. These modifications-a shortening of the red emission wing accompanied by an increase of the emission line intensity-are mainly due to the variation of the radial opacity $\tau_{12}^{r}$ with $\beta_{\infty}$ (via the factor $\left\langle n_{1}\right\rangle$ in eq. [70]) and to the redistribution of photons in a frequency interval which gets narrower and more asymmetrical as $\beta_{\infty}$ is increased. This latter effect is definitely the dominant one, the opacity changes with $\beta_{\infty}$ remaining small. In fact, $\left\langle n_{1}\right\rangle$ does not vary by more than a factor of 3 in the considered $\beta_{\infty}$ range (see Table 3 ). We should also like to point out that for high values of $\beta_{c}$ the maximum of the emission peak may appear redshifted (Fig. $7 h$ ).

Let us now consider the $W_{1}^{R, 0}-W_{1}^{R, 0}$ diagrams (see Fig. 8). By simply reporting in such diagrams the measured moment $W_{1}^{R}$ of an observed $\mathrm{P}$ Cygni line profile, we can derive the parameter $W_{1}^{R, 0}$ and consequently, via equation (68), the fractional mass-loss rate (or a lower limit if the line profile is saturated). This method is especially useful for the analysis of unresolved line profiles. For more details, we refer the reader to our previous papers (Surdej 1983a; Hutsemékers and Surdej 1987, 1989; Surdej and Hutsemékers 1990). In the present application, we have computed line profiles for different values of $W_{1}^{R, 0}$ and via equation (50), their moment $W_{1}^{R}$. The results are reported in Figure $8 a$ for two extreme models. The $W_{1}^{R}$ $-W_{1}^{R, 0}$ curves show a clear dependence on $\beta_{\infty}$, especially for high values of $W_{1}^{R, 0}$, so that we need in principle one diagram for each value of the terminal velocity. Again, this behavior is mainly caused by the emission profile asymmetry. We can nevertheless minimize this dependence by normalizing the curves with respect to the asymptotic value $W_{1}^{R, t}$ of the

TABLE 2

Characteristics of the Relativistic P Cygni Line Profiles Computed for $\beta_{\infty}$ VAlue OF $0.01,0.2,0.5$, AND 0.8

\begin{tabular}{cccc}
\hline \hline Figure & Model & $W_{1}^{R, 0}$ & $\beta_{c} / \beta_{\infty}$ \\
\hline & B. $\boldsymbol{\beta}$ & 1.0 & 0.01 \\
& $\boldsymbol{B} \boldsymbol{\beta}$ & 0.1 & 0.01 \\
& $\boldsymbol{C} \boldsymbol{\beta}$ & 1.0 & 0.01 \\
& $\boldsymbol{B . \epsilon}$ & 1.0 & 0.01 \\
& $\boldsymbol{B .} \boldsymbol{\beta}$ & 1.0 & 0.20 \\
& $\boldsymbol{B .} \boldsymbol{\beta}$ & 1.0 & 0.90 \\
\hline
\end{tabular}

moment calculated in the optically thick approximation, i.e., $W_{1}^{R, 0} \rightarrow \infty$. The values of the moment $W_{1}^{R, t}$ are reported in Table 4 considering the different models. The normalized curves, illustrated in Figure $8 b$, are definitely less affected by the value of $\beta_{\infty}$ so that one diagram of this type may be sufficient to evaluate mass-loss rates with a reasonable degree of accuracy. Normalized diagrams computed for the twelve possible models are illustrated in Figures $9 a-d$. As in the classical limit, the curves are mainly dependent on the opacity distribution.

\section{DISCUSSION AND CONCLUSIONS}

We have learned in the previous sections how it was important to include the relativistic corrections in the radiative

TABLE 3

THE RATIO $\left\langle n_{1}\right\rangle /\left\langle n_{1}\right\rangle_{\text {classical }}$ AS A FUNCTION OF $\beta_{\infty}$ FOR THE DIFFERENT MODELS

\begin{tabular}{|c|c|c|c|}
\hline \multirow[b]{2}{*}{ MODEL } & \multicolumn{3}{|c|}{$\beta_{\infty}$} \\
\hline & 0.2 & 0.5 & 0.8 \\
\hline 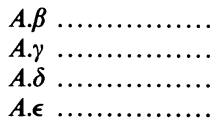 & $\begin{array}{l}0.90 \\
0.94 \\
0.92 \\
0.88\end{array}$ & $\begin{array}{l}0.71 \\
0.85 \\
0.77 \\
0.64\end{array}$ & $\begin{array}{l}0.42 \\
0.74 \\
0.54 \\
0.32\end{array}$ \\
\hline 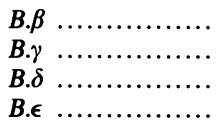 & $\begin{array}{l}0.90 \\
0.94 \\
0.92 \\
0.88\end{array}$ & $\begin{array}{l}0.71 \\
0.86 \\
0.77 \\
0.64\end{array}$ & $\begin{array}{l}0.43 \\
0.76 \\
0.55 \\
0.32\end{array}$ \\
\hline 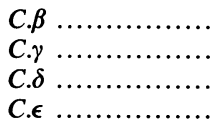 & $\begin{array}{l}0.90 \\
0.95 \\
0.92 \\
0.87\end{array}$ & $\begin{array}{l}0.71 \\
0.87 \\
0.78 \\
0.64\end{array}$ & $\begin{array}{l}0.44 \\
0.80 \\
0.57 \\
0.32\end{array}$ \\
\hline
\end{tabular}

TABLE 4

$\log W_{1}^{R, t}$ AS A FUNCTION OF $\beta_{\infty}$ FOR THE THREE VELOCITY LAWS

\begin{tabular}{cccc}
\hline \hline & \multicolumn{3}{c}{ Velocity LAW } \\
\cline { 2 - 4 }$\beta_{\infty}$ & $A$ & $B$ & $C$ \\
\hline $0.01 \ldots \ldots \ldots$ & -0.31 & -0.32 & -0.35 \\
$0.20 \ldots \ldots \ldots$ & -0.34 & -0.36 & -0.38 \\
$0.50 \ldots \ldots \ldots$ & -0.40 & -0.42 & -0.44 \\
$0.80 \ldots \ldots \ldots$ & -0.47 & -0.49 & -0.51 \\
\hline
\end{tabular}



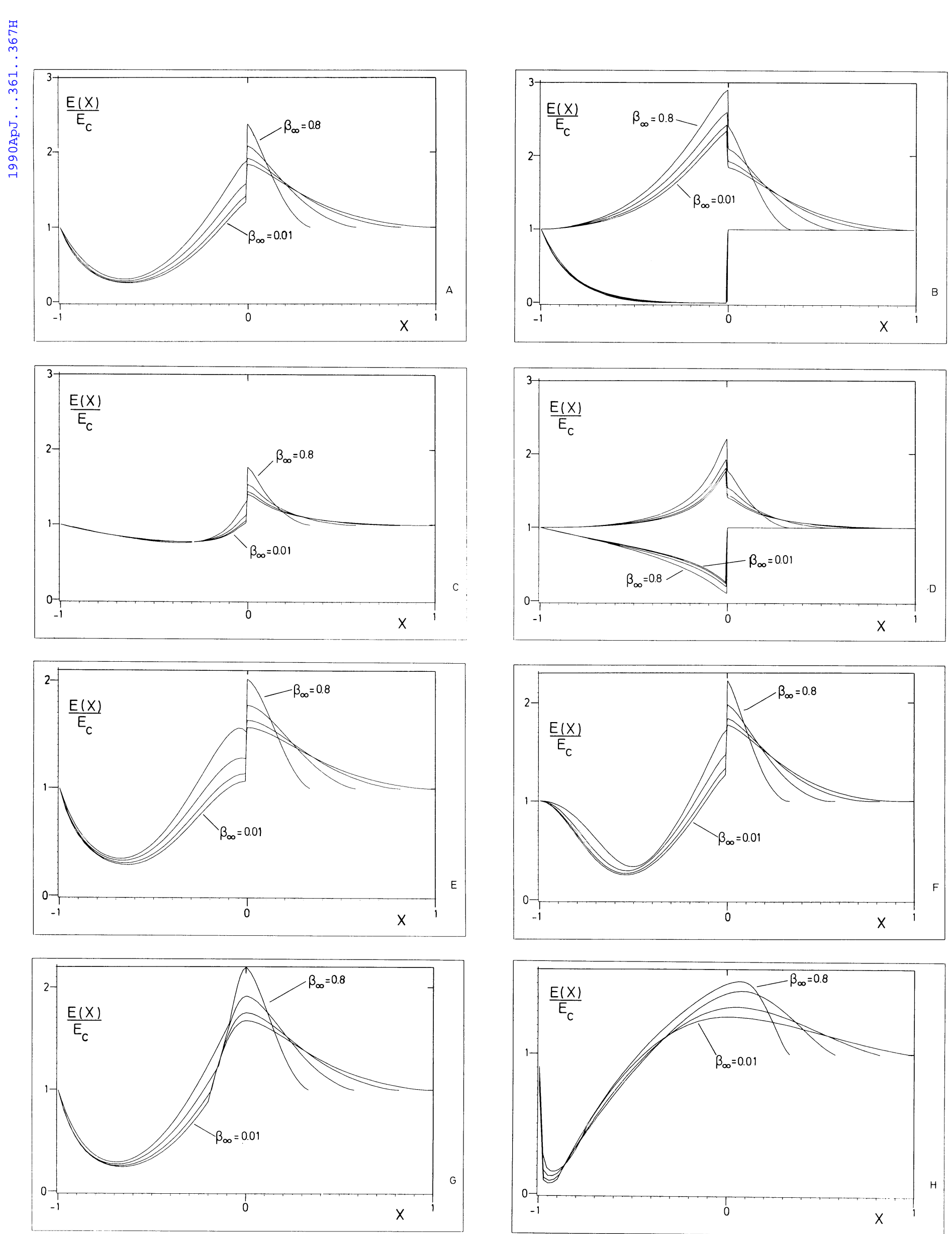

Fig. 7. $-(a-h)$ Examples of relativistic P Cygni line profiles computed for $\beta_{\infty}$ values of $0.01,0.2,0.5$, and 0.8 . Their characteristics are given in Table 2. Figs. $(b)$ and (d) illustrate the absorption and emission components of the profiles shown in $(a)$ and $(c)$. 

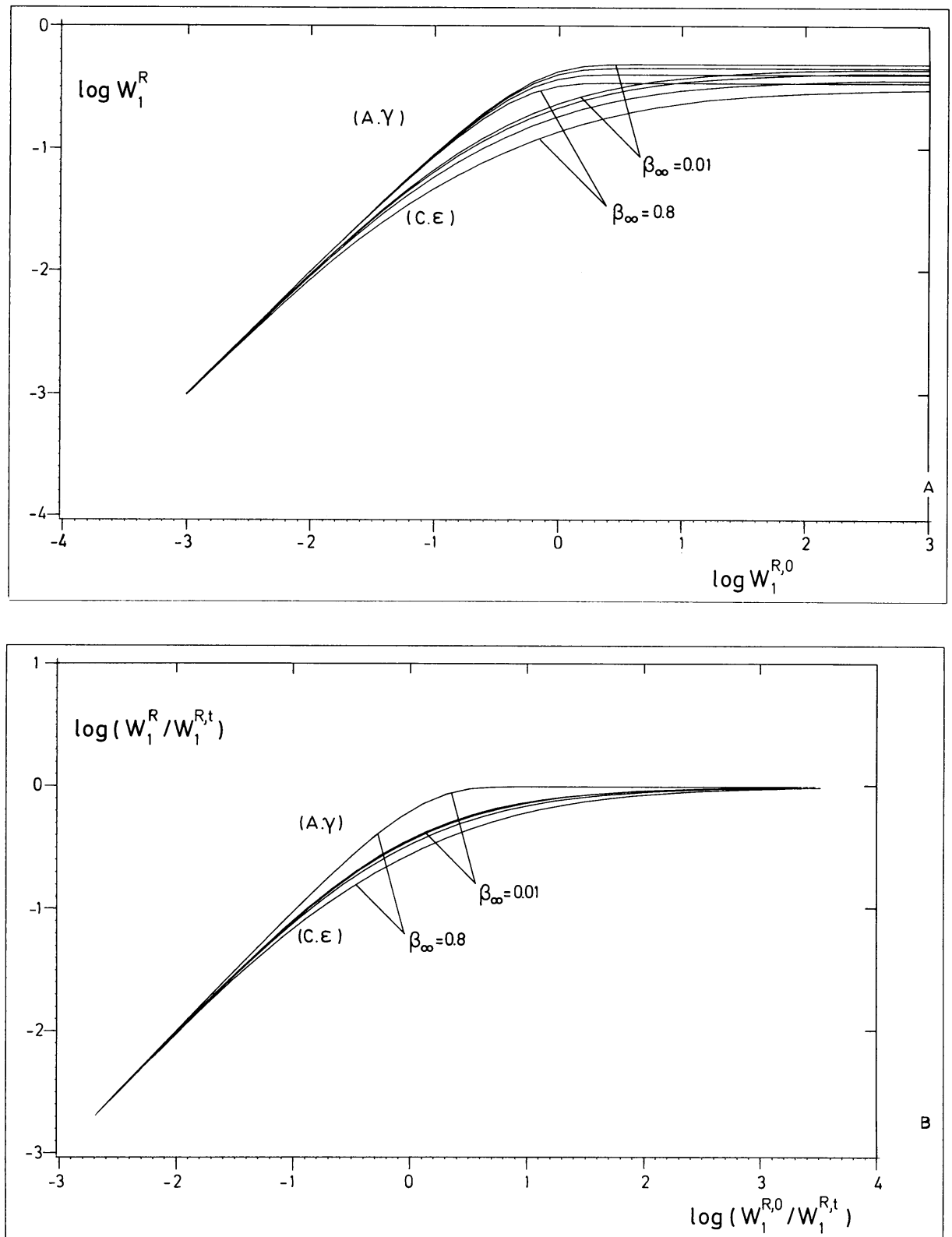

FIG. 8. $-(a-b)$ The first figure illustrates the behavior of the $W_{1}^{R}-W_{1}^{R, 0}$ curves as a function of $\beta_{\infty}$ for two extreme models. As $\beta_{\infty}$ increases, the asymptotic limit $W_{1}^{R, t}$ decreases. The same curves are illustrated in $(b)$ but normalized by the moment $W_{1}^{R, t}$ whose values are given in Table 4.

transfer in order to calculate correctly the source and the line profile functions for large values of $\beta_{\infty}$.

One of the most unexpected effects is certainly the appearance of distant interactions in the envelope when the velocities approach that of light. As in the case of decelerated flows (see Surdej 1978), these interactions may be of great importance when evaluating the amplitude of the radiative forces. Even if they can be neglected most of the time for the type of velocity laws considered here, they can be of much greater importance for other velocity distributions, especially those characterized by very high velocity gradients.

As we have seen, the $\mathbf{P}$ Cygni line profiles formed in relativistically expanding envelopes also suffer important modifications mainly due to the redistribution of photons in a frequency interval whose asymmetry is directly related to the relativistic expression of the Doppler effect. Before comparing the relativistic profiles with the observed ones, it is necessary to rebin the latter ones in a dimensionless frequency scale $(X)$, the asymmetry of the profiles being different on a wavelength scale (i.e., the most extended wing becomes the red one ${ }^{2}$ ). Up to now, the highest expansion velocities $(0.2 c)$ measured from the most extended part of a P Cygni line profile have been reported from the spectra of BAL quasars. For such velocities, the profile modifications remain small compared to the classical case, especially if we remember that other effects, like turbulence, can alter the profiles to a larger extent (see the discussion by

\footnotetext{
${ }^{2}$ More precisely, if we define the dimensionless wavelength $Y=$ $-\left(\lambda-\lambda_{12}^{0}\right) /\left(\lambda_{\min }-\lambda_{12}^{0}\right)$, equivalent to the dimensionless frequency $X$ (eq. [11]) in the classical limit, we easily see that the line profile is formed in the wavelength interval $\left[-1, Y_{\infty}^{R}=1 / X_{\infty}^{R}\right]$ which is greater than the frequency interval given by eq. (13).
} 

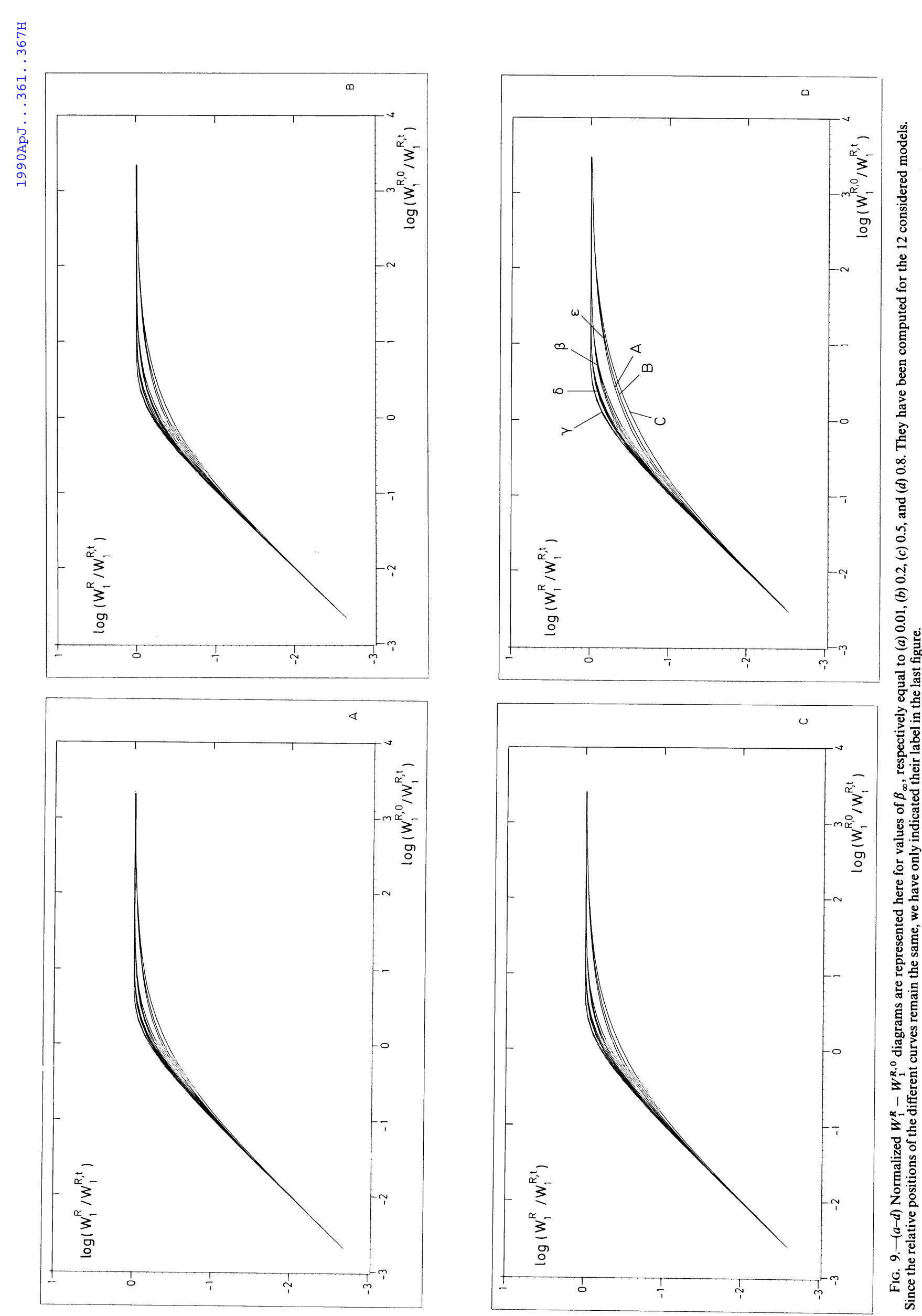

(C) American Astronomical Society - Provided by the NASA Astrophysics Data System 
Surdej and Hutsemékers 1987). Nevertheless, even for $\beta_{\infty}=$ 0.2 , the relativistic corrections do reveal noticeable effects (see Fig. 7).

In spite of the large differences seen for $\beta_{\infty} \geq 0.2$, it is quite surprising that the linear relation between the mass-loss rate and the first-order moment of unsaturated line profiles still holds in the relativistic case. This general relation is therefore independent of many physical and geometrical approximations. Also, the normalized $W_{1}^{R}-W_{1}^{R, 0}$ diagrams only slightly depend on the terminal velocity so that, if the adopted models actually represent realistic physical conditions prevailing in expanding atmospheres, they provide a powerful tool for deriving mass-loss rates. This is especially true from a statistical point-of-view as well as in the context of the analysis of unresolved line profiles.
Throughout this paper, we have introduced limiting approximations. One of the most restrictive is certainly the hypothesis that the opacity and velocity laws representing the expanding envelopes in the classical limit are still adequate in the relativistic case. Only a detailed dynamical analysis could check this hypothesis which is as important as the effects of distant interactions. Due to these approximations, this paper is nothing more than a first step toward a better understanding of the formation of P Cygni line profiles in relativistically expanding atmospheres.

It is a pleasure to thank here the referee for his constructive comments. Part of this work was done while D. H. was a research assistant at the National Funds for Scientific Research (FNRS, Liège Institute of Astrophysics, Belgium).
Beals, C. 1929, M.N.R.A.S., 90, 202.

1931, M.N.R.A.S., 91, 966

Castor, J. I. 1970, M.N.R.A.S., 149, 111.

Castor, J. I., Abbott, D. C., and Klein, R. I. 1975, Ap. J., 195, 157.

Castor, J. I., and Lamers, H. J. G. L. M. 1979, Ap. J. Suppl., 39, 481.

Castor, J. I., Lutz, J. H., and Seaton, M. J. 1981, M.N.R.A.S., 194, 547.

Garmany, C. D., Olson, G. L., Conti, P. S., and van Steenberg, M. E. 1981, Ap. J., 250, 660 .

Hutsemékers, D., and Surdej, J. 1987, Astr. Ap., 173, 101

- 1989, Astr. Ap., 219, 237.

Lucy, L. 1971, Ap. J., 163, 5 .

Mihalas, D. 1978, Stellar Atmospheres (San Francisco: Freeman).

. 1980, Ap. J., 237, 574.

Mihalas, D., and Mihalas, B. W. 1984, Foundations of Radiation Hydrodynamics (Oxford: Oxford University Press).

Olson, G. L. 1981, Ap. J., 245, 1054.

Olson, G. L., and Ebbets, D. 1981, Ap. J., 248, 1021.

Rybicki, G. B., and Hummer, D. G. 1978, Ap. J., 219, 654.

\section{REFERENCES}

Sobolev, V. V. 1958, in Theoretical Astrophysics, ed. V. A. Ambartsumyan (London: Pergamon), chap. 29.

(Ca. 1960, Moving Envelopes of Stars. Transl. by S. Gaposchkin (Cambridge: Harvard University Press).

Surdej, J. 1977, Astr. Ap., 60, 303 (Paper I).

. 1978, Astr. Ap., 62, 135.

. 1979, Astr. Ap., 73, 1 (Paper II)

. 1982, Ap. Space Sci., 88, 31 (Paper III).

$.1983 a$, Astr. Ap., 127, 304.
$-1983 b$, Ap. Space Sci., $90,299$.

Surdej, J., and Hutsemékers, D. 1987, Astr. Ap., 177, 42.

- 1990, Astr. Ap., in press.

Thomas, L. 1930, Quart. J. Appl. Math., 1, 239.

Weiler, K. W., and Sramek, R. A. 1988, Ann. Rev. Astr. Ap., 26, 295.

Weymann, R., and Foltz, C. 1983, in Proc. 24th Liège International Astrophysical Colloquium, Quasars and Gravitational Lenses (Liège: Liegè University Pres), p. 538.

DAMIEN HUTSEMÉKERS: European Southern Observatory, La Silla, Casilla 19001, Santiago 19, Chile

JEAN SURDEJ: Institut d'Astrophysique, Universite de Liège, 5 av. de Cointe, B-4200 Cointe-Ougrée, Belgium 